\title{
HORMÔNIO DO CRESCIMENTO (GH) COM E SEM ATIVIDADE FÍSICA NA BIOQUÍMICA SÉRICA E NO PESO DOS ÓRGÃOS DE RATAS WISTAR.
}

\author{
Marcos Oliveira Santos ${ }^{1}$, Gabriela Azenha Milano Soriano ${ }^{1}$, Caliê Castilho ${ }^{1}$, Cecília Braga Laposy ${ }^{1}$, \\ Francis Lopes Pacagnelli ${ }^{1}$, Rosa Maria Barilli Nogueira ${ }^{1}$, Robson Chacon Castoldi ${ }^{1}$, Rogério \\ Giuffrida ${ }^{1}$, Paula de Carvalho Papa ${ }^{2}$, Hermann Bremmer Neto ${ }^{1}$ e Ines Cristina Giometti ${ }^{1}$ \\ ${ }^{1}$ Universidade do Oeste Paulista - UNOESTE, Presidente Prudente,SP. ${ }^{2}$ Universidade de São Paulo - USP, São Paulo, SP. \\ E-mail: e-mail: inesgiometti@unoeste.br
}

\section{RESUMO}

O objetivo deste trabalho foi verificar o efeito da administração do $\mathrm{GH}$ associado ou não à atividade física na bioquímica sérica e no peso dos órgãos de ratas Wistar. Na presente pesquisa foram utilizadas 40 ratas adultas, divididas em quatro grupos ( $n=10)$ : CT (grupo controle); GH (grupo com administração de GH); Ex (grupo do exercício físico) e ExGH (grupo com exercício físico e com administração de $\mathrm{GH}$ ). Após 30 dias, a bioquímica sérica e o peso dos órgãos foram mensurados. Foi realizada análise de variância (ANOVA), seguida do teste de Tukey. O peso renal do grupo $\mathrm{GH}$ foi maior que Ex e ExGH $(p<0,05)$. A ureia sérica foi menor no grupo ExGH $(p<0,05)$ que nos demais grupos. O grupo Ex apresentou maior dosagem de fosfatase alcalina que os demais. Conclui-se que o $\mathrm{GH}$ na dosagem de $0,2 \mathrm{UI} / \mathrm{Kg}$ por um mês sem a associação com o exercício físico leva a um aumento do peso renal. $\mathrm{O}$ uso do $\mathrm{GH}$ combinado ao exercício físico diminui a ureia sérica, sem alterar a creatinina. A atividade física sem a administração de $\mathrm{GH}$ aumenta a fosfatase alcalina.

Palavras-chave: lipídeos, treinamento físico, rim, peso dos órgãos, Rattus novergicus.

\section{GROWTH HORMONE (GH) WITH AND WITHOUT PHYSICAL ACTIVITY IN SERUM BIOCHEMISTRY AND ORGAN WEIGHTS IN WISTAR RATS.}

\begin{abstract}
The objective of this study was to evaluate the effect of $\mathrm{GH}$ administration with or without physical activity in serum biochemistry and organ weights in Wistar rats. In this study were used 40 adult female rats were divided into four groups $(n=10)$ CT (control group); GH (GH administration with group); Ex (the exercise group) and ExGH (group exercise and $\mathrm{GH}$ administration). After 30 days, serum biochemistry and organ weights were measured. It performed analysis of variance (ANOVA) followed by Tukey's test. The kidney weight of group was greater than $G H$ and Ex ExGH $(p<0.05)$. Serum urea was lower in ExGH group $(p<0.05)$ than the other groups. The former group had higher dosage alkaline phosphatase than the others. It is concluded that $\mathrm{GH}$ at dose of $0.2 \mathrm{IU} / \mathrm{kg}$ for one month without combination with physical exercise leads to an increase in kidney weight. The use of $\mathrm{GH}$ combined with exercise reduces serum urea, without changing the creatinine. The physical activity without GH administration increases alkaline phosphatase.
\end{abstract}

Keywords: lipids, physical training, kidney, organ weights, Rattus norvegicus. 


\section{INTRODUÇÃO}

O hormônio do crescimento $(\mathrm{GH})$ é um polipeptídeo de 22 KDa secretado pela hipófise anterior, é requerido para o crescimento longitudinal e certos aspectos do metabolismo global (CARTER-SU; SCHWARTZ; SMIT, 1996). O GH promove o crescimento e o aumento de todos os tecidos do corpo até a maturação (WILMORE; COSTILL, 2001). Os principais efeitos do GH são o aumento da síntese proteica, a diminuição da degradação de proteínas, o aumento da mobilização de lipídeos, a diminuição da oxidação de glicose e o aumento do armazenamento de glicogênio (KOPPLE, 1992). O GH pode diretamente exercer seus efeitos nas células alvo, ou suas ações podem ser mediadas através do fator de crescimento semelhante à insulina (IGF), também conhecido como somatomedina C (PARK; VANDERHOOF, 1996). O GH age diretamente sobre as células do fígado, ligando-se ao seu receptor (GHR) e induzindo uma série de eventos que acabam resultando, por exemplo, na produção do IGF-1 (VANCE, 1990). Os GHRs foram demonstrados em diversas células, incluindo queratinócitos, fibroblastos e células endoteliais (LOBIE et al., 1990). Os IGFs são sintetizados pelo fígado, e pela maioria das células orgânicas, quando estimulados pelo GH e são divididos em IGF-1 e IGF-2 (LOBIE et al., 1990).

Durante o exercício físico ocorre constante liberação de $\mathrm{GH}$, e esta liberação aumenta conforme aumenta o exercício (DEUSCHLE et al., 1998). O GH durante o exercício físico aumenta mais nas pessoas não condicionadas e declina mais rapidamente nas pessoas condicionadas (WILMORE; COSTILL, 2001).

A busca incessante pelo melhor desempenho atlético ou corpo esteticamente perfeito tem levado muitas mulheres a além de praticar atividades físicas, utilizar produtos, indevidamente, sem prescrição médica. São vários os produtos utilizados para esse fim, como o GH. O uso do GH aumenta a massa muscular, por isso, há o relato do uso abusivo deste hormônio por atletas para melhorar o desempenho (CLARKSON, 1991).

$\mathrm{O}$ uso indiscriminado do $\mathrm{GH}$ pode acarretar danos à saúde do indivíduo, causando mudanças metabólicas que estão associadas com efeitos colaterais deletérios como instabilidade cardíaca, hipertensão, resistência à insulina, acromegalia (RENNIE, 2003). Além disso, não há evidências concretas de um efeito anabólico em humanos adultos saudáveis (RENNIE, 2003).

Por tudo isso relatado, o objetivo deste trabalho foi verificar o efeito da administração do GH associado ou não à atividade física na bioquímica sérica e no peso dos órgãos de ratas Wistar. 


\section{MATERIAL E MÉTODOS}

Na presente pesquisa foram utilizadas 40 ratas, com idade de 9 semanas, provenientes do Biotério da UNOESTE- Universidade do Oeste Paulista. As ratas foram mantidas em 12 caixas com 3 a 4 animais cada com marcação individual, recebendo água e ração da marca Supralab ${ }^{\circledR} a d$ libitum, com temperatura ambiente entre $20-30^{\circ} \mathrm{C}$ e luz controlada em ciclo de 12 horas (claro e escuro).

Os animais foram aleatoriamente divididos em quatro grupos $(n=10)$ : CT (grupo controle sem exercício físico e sem administração de $\mathrm{GH}$ ); $\mathrm{GH}$ (grupo sem exercício físico e com administração de GH); Ex (grupo com exercício físico e sem administração de GH) e ExGH (grupo com exercício físico e com administração de GH).

As ratas submetidas ao uso de 0,2 UI/Kg de hrGH subcutâneo seguindo protocolo de Kaminsky et al. (2012), da marca Saizen ${ }^{\circledR}$, o medicamento foi aplicado a cada 2 dias, por 30 dias (segunda-feira, quarta-feira e sexta-feira), utilizando uma seringa de insulina. Nas demais ratas, a solução fisiológica foi administrada em volume similar.

O exercício físico foi realizado por meio de um protocolo de exercício muscular de saltos verticais na água segundo Malheiro et al. (2009). Uma semana antes de iniciar o experimento, as ratas foram adaptadas ao exercício físico na água. O treinamento de adaptação consistia em realizar séries progressivas (uma, duas e três) de 10 saltos verticais com coletes acomodados na região anterior ao tórax que continham uma sobrecarga de $50 \%$ do peso corporal, a cada dois dias, dentro de um tubo de PVC de $25 \mathrm{~cm}$ de diâmetro $\mathrm{com} 38 \mathrm{~cm}$ de água aquecida $\left(30^{\circ} \mathrm{C}\right)$ em seu interior. Os animais eram pesados em um a cada dois dias de exercício físico a fim recalcular a carga do colete. Após esta adaptação, os animais foram submetidos a um protocolo de 4 séries de 10 saltos com intervalo de 1 minuto entre cada série, três vezes por semana, durante 1 mês. Após a prática da atividade física era realizada a secagem das ratas.

Ao final de um mês, as ratas foram pesadas, medidas, anestesiadas com éter etílico, para não influenciar nos parâmetros bioquímicos, e então foram sacrificadas por exsanguinação. Foi realizada colheita de sangue para bioquímica sérica da glicose, enzimas hepáticas (AST - aspartato transaminase e ALT - alanina transaminase), ureia, creatinina, fosfatase alcalina (FA), colesterol e triglicerídeos em tubos em tubos (Vacutainer ${ }^{\oplus}$ ) sem anticoagulante, e com fluoreto para a dosagem de lactato. A bioquímica sérica foi realizada por meio do método colorimétrico utilizando sistemas Cobas $\mathrm{C} 111^{\circledR}$. Foram verificados os pesos em balança de precisão do fígado, coração, útero, ovários, baço e rins, para verificação se o GH iria interferir na morfometria dos animais. 
Todos os resultados foram analisados quanto ao pressuposto de normalidade, empregando-se o teste de Shapiro-Wilk $(p>0,05)$. Todas as variáveis foram submetidas ao teste análise de variância (ANOVA) para comparar as médias dos quatro grupos, seguida do teste de Tukey. Todas as análises estatísticas foram realizadas empregando-se os programas de Bioestat 5.0 ${ }^{\oplus}$ e GraphPad InStat versão 3.0. O nível de significância adotado para todas as comparações foi de $5 \%$.

Este experimento foi aprovado pela Comissão de Ética no Uso de Animais (CEUA) Institucional (número do processo: 1014).

\section{RESULTADOS}

Os valores referentes aos órgãos das ratas estão representados na Tabela 1 . Não houve diferença entre os grupos na média de peso dos seguintes órgãos, fígado, adrenal, ovários, baço e útero $(P>0,05)$ após 30 dias de experimento. Houve diferença estatística significativa na média de peso dos rins, os grupos com atividade física apresentaram um menor peso renal que o grupo GH.

Tabela 1 - Médias e desvios-padrão referente ao peso de órgãos das ratas, após o período de quatro semanas de experimento.

\begin{tabular}{lllll}
\hline morfometria (g) & CT & GH & Ex & ExGH \\
\hline Rim & $1,57 \pm 0,10^{\mathrm{ab}}$ & $1,68 \pm 0,11^{\mathrm{a}}$ & $1,49 \pm 0,14^{\mathrm{b}}$ & $1,53 \pm 0,12^{\mathrm{b}}$ \\
Fígado & $8,39 \pm 3,09^{\mathrm{a}}$ & $9,96 \pm 0,69^{\mathrm{a}}$ & $9,13 \pm 1,23^{\mathrm{a}}$ & $8,89 \pm 0,82^{\mathrm{a}}$ \\
Adrenal & $0,10 \pm 0,02^{\mathrm{a}}$ & $0,10 \pm 0,01^{\mathrm{a}}$ & $0,14 \pm 0,10^{\mathrm{a}}$ & $0,10 \pm 0,02^{\mathrm{a}}$ \\
Ovário & $0,16 \pm 0,24^{\mathrm{a}}$ & $0,08 \pm 0,01^{\mathrm{a}}$ & $0,16 \pm 0,22^{\mathrm{a}}$ & $0,09 \pm 0,02^{\mathrm{a}}$ \\
Baço & $0,51 \pm 0,06^{\mathrm{a}}$ & $0,57 \pm 0,10^{\mathrm{a}}$ & $0,52 \pm 0,07^{\mathrm{a}}$ & $0,49 \pm 0,06^{\mathrm{a}}$ \\
Útero & $0,45 \pm 0,05^{\mathrm{a}}$ & $0,49 \pm 0,07^{\mathrm{a}}$ & $0,45 \pm 0,05^{\mathrm{a}}$ & $0,43 \pm 0,11^{\mathrm{a}}$ \\
\hline
\end{tabular}

CT (animais sem exercício físico e submetidos e sem administração de hormônio), GH (animais sem exercício físico e com administração do hormônio do crescimento), Ex (animais submetidos ao exercício físico e sem administração de hormônio), ExGH (animais submetidos à atividade física e à aplicação de hormônio do crescimento). Valores na mesma linha seguidos de letras minúsculas diferentes diferem estatisticamente $(p>0,05)$.

Com relação aos parâmetros bioquímicos verificados nos animais, houve diferença significativa na dosagem de ureia, o grupo ExGH apresentou menor valor de ureia que os demais grupos (Tabela 2). A dosagem de fosfatase alcalina também apresentou diferença entre os grupos, sendo que o maior valor foi no grupo Ex diferindo significativamente dos outros grupos (Tabela 2). 
Tabela 2 - Médias e desvios-padrão referente a analise dos componentes do sangue das ratas, após o período de quatro semanas de experimento.

\begin{tabular}{lllll}
\hline Parâmetros & CT & GH & Ex & ExGH \\
\hline Glicose (mg/dL) & $133,56 \pm 17,74^{\mathrm{a}}$ & $124,80 \pm 17,02^{\mathrm{a}}$ & $144,30 \pm 27,47^{\mathrm{a}}$ & $143,44 \pm 11,35^{\mathrm{a}}$ \\
AST (UI/L) & $127,21 \pm 87,09^{\mathrm{a}}$ & $145,56 \pm 72,49^{\mathrm{a}}$ & $100,06 \pm 18,30^{\mathrm{a}}$ & $106,17 \pm 26,61^{\mathrm{a}}$ \\
ALT (UI/L) & $76,75 \pm 9,22^{\mathrm{a}}$ & $80,56 \pm 12,82^{\mathrm{a}}$ & $80,00 \pm 20,65^{\mathrm{a}}$ & $75,33 \pm 10,28^{\mathrm{a}}$ \\
Ureia (mg/dL) & $55,88 \pm 5,48^{\mathrm{b}}$ & $56,56 \pm 8,95^{\mathrm{b}}$ & $57,55 \pm 4,96^{\mathrm{a}}$ & $47,61 \pm 4,46^{\mathrm{b}}$ \\
Creatinina (mg/dL) & $0,66 \pm 0,26^{\mathrm{a}}$ & $0,54 \pm 0,05^{\mathrm{a}}$ & $0,61 \pm 0,04^{\mathrm{a}}$ & $0,59 \pm 0,06^{\mathrm{a}}$ \\
FA (UI/L) & $103,36 \pm 36,61^{\mathrm{b}}$ & $113,63 \pm 33,11^{\mathrm{b}}$ & $195,22 \pm 72,25^{\mathrm{a}}$ & $106,94 \pm 21,42^{\mathrm{b}}$ \\
Lactato (mg/dL) & $56,76 \pm 17,42^{\mathrm{a}}$ & $55,60 \pm 15,37^{\mathrm{a}}$ & $59,54 \pm 15,81^{\mathrm{a}}$ & $53,49 \pm 19,45^{\mathrm{a}}$ \\
Colesterol (mg/dL) & $89,29 \pm 16,53^{\mathrm{a}}$ & $96,00 \pm 24,50^{\mathrm{a}}$ & $95,30 \pm 17,07^{\mathrm{a}}$ & $92,59 \pm 23,06^{\mathrm{a}}$ \\
Triglicerídeos (mg/dL) & $129,00 \pm 52,09^{\mathrm{a}}$ & $110,80 \pm 50,20^{\mathrm{a}}$ & $130,50 \pm 36,47^{\mathrm{a}}$ & $121,89 \pm 25,51^{\mathrm{a}}$ \\
\hline
\end{tabular}

CT (animais sem exercício físico e submetidos e sem administração de hormônio), GH (animais sem exercício físico e com administração do hormônio do crescimento), Ex (animais submetidos ao exercício físico e sem administração de hormônio), ExGH (animais submetidos à atividade física e à aplicação de hormônio do crescimento), AST (aspartato transaminase), ALT (alanina transaminase), CK (creatina quinase), CK-MB (creatina quinase do músculo-cérebro), FA (fosfatase alcalina). Valores na mesma linha seguidos de letras minúsculas diferentes diferem estatisticamente $(p>0,05)$.

\section{DISCUSSÃO}

Quando se avaliou o peso dos principais órgãos, apenas os rins mostraram diferença entre os grupos, as ratas que não praticaram exercício físico e receberam GH apresentaram maior peso dos rins que as ratas que praticavam exercício físico independente da administração do $\mathrm{GH}$, este resultado difere do que foi apresentado na pesquisa de Rosenbloom (ROSENBLOOM, 2009), onde pessoas com problemas de crescimento tratados com GH apresentaram aumento de todo o corpo e dos órgãos. Porém, os animais do presente trabalho não apresentavam patologias que comprometessem o crescimento.

O comprometimento funcional dos rins é determinado a partir do valor da creatinina sérica ou do valor estimado pela coleta do "Clearance" de creatinina (OLIVEIRA et al., 2008). Apesar de o grupo GH apresentar maior peso renal no presente trabalho, não foi verificada diferença significativa na creatinina sérica entre os grupos.

Não foi encontrada diferença na porcentagem de triglicerídeos ou colesterol. Outros autores, trabalhando com outras metodologias citam diminuição de gordura corporal com a administração de GH. Fett e Rezende (2001) relatam que o aumento de GH tem grande influência no ganho de massa muscular, força e queima de gordura, especialmente naqueles indivíduos que recebem suplementação com GH. Oliveira et al. (2008) relatam que as alterações características na deficiência desse hormônio na composição corporal incluem redução da quantidade de massa 
magra (kg) e aumento do percentual de gordura. Já a pesquisa de Halpern et al. (HALPERN et al., 2006) conclui que obesos tratados com GH por três meses apresentaram melhora do perfil lipídico. Cruzat et al. (2008) sugerem que o consumo de GH mobiliza ácidos graxos livres do tecido adiposo para geração de energia aumentando a capacidade de oxidação de gordura e gerando um maior gasto energético.

Esperava-se no presente trabalho que o GH levasse a uma alteração bioquímica e a um aumento dos órgãos devido ao aumento de IGF-1 local, porém isso não foi observado. Pode ser que os hormônios femininos das ratas tenham influenciado neste resultado. $O$ tratamento com estrógenos orais, concomitante ao uso de GH em pacientes com hipopituitarismo, antagoniza as ações biológicas do GH. O estrógeno oral inibe a secreção/produção de IGF-1 por meio de efeito de primeira passagem hepática, causando aumento da secreção/produção de GH por intermédio de inibição do "feedback" negativo de IGF-1 em mulheres normais (ISOTTON; WENDER; CZEPIELEWSKI, 2008). Além disso, durante o exercício físico constante, a liberação do GH é estimulada e a quantidade deste hormônio liberada é tanto maior quanto mais intenso for o exercício. Isto ocorre porque o exercício físico estimula a produção de opiáceos endógenos, que inibem a produção de somatostatina hepática, um hormônio que reduz a liberação de GH (DEUSCHLE et al., 1998; MCARDLE; KATCH; KATCH, 2003).

O presente trabalho encontrou diferença estatística na ureia e fosfatase alcalina analisada no final do experimento. O grupo que recebeu a aplicação de GH e realizou os saltos na água, apresentou-se com menor valor de ureia que os outros grupos. A concentração sérica de ureia sem concomitante redução de creatinina pode estar correlacionada ao metabolismo de proteínas. Em estudo de Fayh et al. (2007) o GH levou a hipertrofia muscular pela facilitação do transporte de aminoácidos para o interior da célula, estimulando o metabolismo de gorduras. Na dosagem de fosfatase alcalina, o grupo de animais com atividade física e sem hormônio apresentou um valor maior que os outros grupos.

\section{CONCLUSÃO}

Conclui-se que a utilização de GH na dosagem de 0,2UI/Kg por um mês sem a associação com o exercício físico leva a um aumento do peso renal. O uso do GH combinado à atividade física diminui a ureia sérica, sem alterar a creatinina. A atividade física sem a administração de GH aumenta a fosfatase alcalina. 


\section{REFERÊNCIAS}

CARTER-SU, C; SCHWARTZ, J; SMIT, L S. Molecular mechanism of growth hormone action. Annual review of physiology v. 58, p. 187-207, Jan. 1996.

CLARKSON, P M. Nutritional ergogenic aids: chromium, exercise, and muscle mass. International journal of sport nutrition v. 1, n. 3, p. 289-293, 1991.

CRUZAT, Vinicius Fernandes et al. Hormônio do crescimento e exercício físico: considerações atuais .Revista Brasileira de Ciências Farmacêuticas. [S.I: s.n.]. , 2008

DEUSCHLE, $M$ et al. Endurance training and its effect upon the activity of the GH-IGFs system in the elderly. International journal of sports medicine v. 19, n. 4, p. 250-254, 1998.0172-4622 (Print)r0172-4622 (Linking).

FAYH, Ana Paula Trussardi et al. Efeito da suplementação de L-arginina sobre a secreção de hormônio do crescimento e fator de crescimento semelhante à insulina em adultos .Arquivos Brasileiros de Endocrinologia \& Metabologia. [S.I: s.n.]. , 2007.

FETT, Carlos Alexandre; REZENDE, Waléria Christiane. A termogênese como recurso de intervenção eficiente para o combate e controle da obesidade. R Min Educ Fis v. 9, n. 1, p. 83-106, 2001.

Disponível em: <http://www.revistamineiradeefi.ufv.br/artigos/arquivos/a7efe350c30de53bfde50e701044e6db.p $\mathrm{df}>$.

FRISCH, H. Growth hormone and body composition in athletes. Journal of Endocrinological Investigation v. 22, n. 5 SUPPL., p. 106-109 , 1999.0391-4097 (Print)r0391-4097 (Linking).

HALPERN, Alfredo et al. Efeito do hormônio de crescimento sobre parâmetros antropométricos e metabólicos na obesidade andróide .Arquivos Brasileiros de Endocrinologia \& Metabologia. [S.I: s.n.]. , 2006.

ISOTTON, Ana Lúcia; WENDER, Maria Celeste O.; CZEPIELEWSKI, Mauro A. Influências da reposição de estrógenos e progestágenos na ação do hormônio de crescimento em mulheres com hipopituitarismo .Arquivos Brasileiros de Endocrinologia \& Metabologia. [S.I: s.n.]. , 2008.

KAMINSKY, P. et al. Growth hormone potentiates thyroid hormone effects on post-exercise phosphocreatine recovery in skeletal muscle. Growth Hormone and IGF Research v. 22, p. 240244 , 2012. http://dx.doi.org/10.1016/i.ghir.2012.08.001

KOPPLE, J D. The rationale for the use of growth hormone or insulin-like growth factor I in adult patients with renal failure. Mineral and electrolyte metabolism v. 18, n. 2-5, p. 269-275, 1992.0378-0392 (Print) 0378-0392 (Linking).

LOBIE, P. E. et al. Localization of the growth hormone receptor/binding protein in skin. Journal of Endocrinology v. 126, n. 3, p. 467-472, 1990. http://dx.doi.org/10.1677/joe.0.1260467

MALHEIRO, O. C D M et al. Calcaneal tendon regions exhibit different MMP-2 activation after vertical jumping and treadmill running. Anatomical Record v. 292, p. 1656-1662 , 2009.1932-8494 (Electronic)r1932-8486 (Linking). 
MCARDLE, W D; KATCH, F I; KATCH, V L. Fisiologia do exercício energia, nutrição e desempenho humano. 5. ed. Rio de Janeiro: Guanabara Koogan, 2003.

OLIVEIRA, Josenilson Campos De et al. Baixa estatura na doença renal crônica: fisiopatologia e tratamento com hormônio de crescimento .Arquivos Brasileiros de Endocrinologia \& Metabologia. [S.I: s.n.]. , 2008.

PARK, J H; VANDERHOOF, J A. Growth hormone did not enhance mucosal hyperplasia after smallbowel resection. Scandinavian journal of gastroenterology v. 31, n. 4, p. 349-354, 1996.00365521 (Print)r0036-5521 (Linking).

RENNIE, M J. Claims for the anabolic effects of growth hormone: a case of the emperor's new clothes? British journal of sports medicine v. 37, n. 2, p. 100-5 , Apr. 2003.

ROSENBLOOM, Arlan L. Mecasermin (recombinant human insulin-like growth factor I).Advances in Therapy. [S.I: s.n.]. , 2009.

VANCE, M L. Growth hormone for the elderly? .The New England journal of medicine. [S.I: s.n.]. , 1990.

WILMORE, J H; COSTILL, D L. Physical energy: fuel metabolism. Nutrition reviews v. 59, n. 1 Pt 2, p. S13-6, Jan. 2001. 